\title{
Editorials
}

\section{New NICE guidance on diagnosing cancer in general practice}

In June 2015 the National Institute for Health and Care Excellence (NICE) published its updated guidance on suspected cancer, replacing the previous 2005 guidelines, which had underpinned the '2-week wait' (2WW) referral system in England and Wales.' These guidelines, heralded as potentially saving 5000 lives per year in England, ${ }^{2}$ are just one aspect of a wide-ranging set of government policies over the past 15 years aimed at improving cancer outcomes.

\section{NEW CANCER RISK THRESHOLDS}

There are important changes in the new guidance. Perhaps most significant is that they are much better grounded on epidemiological evidence from primary care, rather than the old guidelines that relied predominantly on secondary care data. This new evidence enabled the guideline developers to identify the patterns of symptoms, signs, and simple investigations associated with specific levels of risk of an undiagnosed cancer. As a consequence, the guidelines have established explicit risk thresholds to recommend urgent investigation within 2 weeks. The old guidelines recommended urgent investigation for symptoms associated with a disparate range of cancer risks but few symptoms had a risk of $<5 \%$. In the new guidance a risk threshold of 3\% was set for adult cancers, and lower risks agreed for children and young adults who potentially have more to gain from early diagnosis. This was a pragmatic decision balancing patient viewpoints, which in a large English study suggested a preferred $1 \%$ threshold, ${ }^{3}$ and the economic and clinical costs of investigating large numbers of people at low risk of cancer. However, it is important to recognise how the underlying evidence could overestimate the actual risk of cancer as GPs may only record symptoms when they suspect them to be significant. In addition, symptoms in general practice present along a continuum: abdominal pain, bowel habits, or cough are not simply on or off.

Advances in the epidemiological evidence have allowed the guidelines to extend beyond predominantly single symptoms to recognise the importance of combinations of symptoms in predicting risk of cancer. The guidelines also account better for age and smoking as the most important underlying risk factors when considering certain common symptoms. For example, someone aged $>40$ years with abdominal pain and

the guidelines have established explicit risk thresholds to recommend urgent investigation within 2 weeks ... a risk threshold of $3 \%$ was set for adult cancers, and lower risks agreed for children and young adults who potentially have more to gain from early diagnosis.

weight loss should be investigated urgently for colorectal cancer. If they are aged $>60$ years, they should also be investigated for pancreatic cancer by CT or ultrasound. ${ }^{4}$

\section{IMPROVING DIRECT ACCESS TO INVESTIGATIONS}

The new guidance also recommends extending direct access to a range of diagnostic tests including CT lsuspected pancreatic), MRI (suspected brain), and upper GI endoscopy (suspected oesophageal or stomach cancer). Giving GPs direct access to and responsibility for investigations in the initial pathway supports the pivotal role of general practice in raising the suspicion of cancer. ${ }^{5}$ Furthermore, this decision recognises the national variation in access to these tests and the potential to reduce diagnostic intervals through direct access pathways. ${ }^{6}$ NICE conducted a healtheconomic analysis to inform decisions about diagnostic tests on people with symptoms of bowel cancer which fall below the 3\% threshold. The analysis compared guaiacbased faecal occult blood test (FOBT), faecal immunochemical test (FIT), barium enema, CT colonography, and colonoscopy. At the $€ 20000$ per QALY threshold FOBT is the most cost-effective test for people with lower risk symptoms (1-3\%). On this basis the guidance recommends FOBT, for example, in people aged $<60$ years with a change in bowel habit. This is a significant change in recommended practice and provides a strong rationale for using FOBT beyond its current indication as a screening test in asymptomatic populations. However, the evidence on using FOBT or FIT on symptomatic patients in general practice is relatively scarce and primary care-based research on the effectiveness of direct access to such investigations is needed. Falsenegative tests could potentially postpone a referral for a more accurate investigation.

\section{NON-SPECIFIC SYMPTOMS OF CANCER}

Previous NICE guidelines were presented only in a tumour-specific way, but the new guidance is also summarised by symptoms, recognising that certain common symptoms are associated with more than one cancer. Importantly, there is a section relating to non-specific features of cancer including appetite loss, weight loss, and fatigue. Weight loss is associated with a $7 \%$ overall risk of cancer but this includes colorectal, gastrooesophageal, lung, prostate, pancreatic, and urological cancers. Weight loss is of course associated with a range of other serious non-malignant conditions and this is where clinical acumen is important in determining the most appropriate line of investigation, including selecting a specific cancer pathway. In Denmark, diagnostic centres have been established for patients with such non-specific but serious symptoms and show a $15 \%$ risk of cancers among the referred patients, most often lung, colorectal, and haematological cancers. ${ }^{7}$ This avoids the time-consuming need to define a specific cancer pathway for urgent investigation and allows assessment by several different clinical specialities in a single visit. ${ }^{8}$ Pilot sites of diagnostic centres are currently being established in England as part of the Accelerate, Coordinate, and Evaluate (ACE) programme in earlier cancer diagnosis as an alternative pathway for people with nonspecific serious symptoms.

\section{PATIENT COMMUNICATION}

The importance of good patient communication is emphasised in the guidelines, highlighting two specific areas. The first is safety netting as an approach to manage low-risk non-specific symptoms which may be early symptoms of cancer. The majority of such symptoms in primary care resolve spontaneously but their persistence 


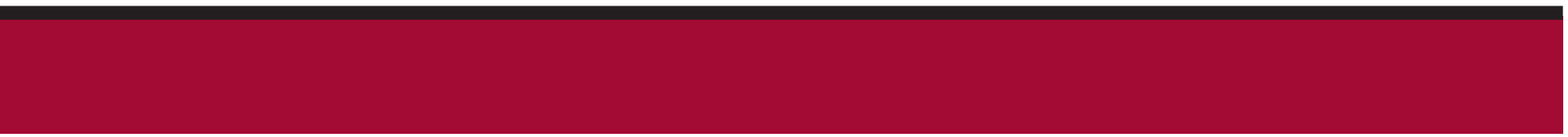

\section{"If the NICE guidelines are implemented successfully they could increase timely cancer diagnosis and, more importantly, reduce disparities in cancer survival between England and other developed nations.}

is associated with increased risk of cancer. Patients should be given clear guidance about time frames in which to return if their symptoms persist or worsen, or new symptoms develop. But although this is one of the core strategies in general practice, the structure and effect of safety netting has not been well studied and needs further development and testing.

The second aspect of patient communication relates to information and support at the point of referral. Many patients are unaware they have been referred for suspected cancer or to a cancer service. The new guidelines recommend that GPs discuss with their patient why they are referring them to a cancer service, while trying to reassure them that they are unlikely to have cancer.

\section{IMPLEMENTING THE NEW GUIDELINES}

The major challenge for these new guidelines is how to implement them across the health service. The previous NICE guidelines focused on single symptoms but GPs are now faced with a more complex set of rules to determine cancer risk. Macmillan Cancer Support has developed a cancer decision support tool (CDS) which is integrated into GP clinical software and is currently being distributed widely. The CDS applies Hamilton's tumourspecific risk models and the symptom-based QCancer ${ }^{\circledR}$ risk models. ${ }^{9}$ Further development and evaluation of this software could be critical for successful implementation of the NICE guidance, although many questions remain about how best to use this type of CDS in practice, especially during the consultation. ${ }^{10}$ When the previous NICE guidelines were implemented, it required major changes in hospital outpatient services to meet the 2-week targets. Their implementation was associated with an overall reduction in the mean diagnostic interval (time from presentation in general practice to diagnosis) for all cancers, not only those referred urgently, of 5.4 days. ${ }^{11}$ Significant reductions were observed for colorectal, pancreatic, oesophageal, renal, bladder, and head and neck cancers but cancers such as lung, myeloma, and lymphoma continued to have long median diagnostic intervals of over 100 days. On average $11 \%$ of people referred via the $2 \mathrm{WW}$ pathway are found to have cancer (the 'conversion rate') but only $43 \%$ of cancers are referred along this route. ${ }^{12}$ Concerns have been raised about the impact of lowering the referral threshold on the volume of referrals, especially to radiology and endoscopy services, and the likely reduced conversion rate. The NICE Costing Statement recognises that commissioners should increase investment in these diagnostic services and suggests this will be balanced by reduced emergency presentations and lower costs of treating earlier stage disease. But when implementing these NICE guidelines the evidence and reasoning behind this costing will have to be understood and supported by commissioners, and hospital consultants and administrators.

The new NICE guidelines represent the first national approach to reduce 'diagnostic delay' by systematically applying evidence on how cancer presents in primary care. Guidelines on 'specific pathways for specific symptoms for specific cancers' will never be enough to account fully for the clinical reality seen by the GP responsible for managing the risk of serious disease in the front line of our health system. The International Cancer Benchmarking Partnership Module 3 study suggests that GPs in England and Wales have higher thresholds to investigate people with cancer symptoms than GPs from Australia and Canada, where cancer survival rates are better. ${ }^{13}$ If the NICE guidelines are implemented successfully they could increase timely cancer diagnosis and, more importantly, reduce disparities in cancer survival between England and other developed nations. ${ }^{14}$

\section{Jon Emery,}

Herman Professor of Primary Care Cancer Research, NHMRC Practitioner Fellow, Department of General Practice, University of Melbourne, Melbourne, Australia.

\section{Peter Vedsted,}

Director, Research Centre for Cancer Diagnosis in

Primary Care, Institute for Public Health, Aarhus University, Aarhus, Denmark

\section{Provenance}

Commissioned; not externally peer reviewed.

DOI: 10.3399/bjgp15X686401

\section{ADDRESS FOR CORRESPONDENCE}

Jon Emery

Department of General Practice, University of

Melbourne, 200 Berkeley Street, Carlton, Victoria 0053, Australia

\section{E-mail: jon.emeryæunimelb.edu.au}

\section{REFERENCES}

. National Institute for Health and Care Excellence. Suspected cancer: recognition and referral. NG12. London: NICE, 2015.

2. Abdel-Rahman $M$, Stockton $D$, Rachet $B$, et al. What if cancer survival in Britain were the same as in Europe: how many deaths are avoidable? Br J Cancer 2009; 101(Suppl 2): S115-S124.

3. Banks J, Hollinghurst S, Bigwood L, et al. Preferences for cancer investigation: a vignettebased study of primary-care attendees. Lancet Oncology 2014; 15(2): 232-240.

4. Hamilton W, Hajioff S, Graham J, SchmidtHansen M. Suspected cancer lpart 2-adults): reference tables from updated NICE guidance. BMJ 2015; 350: h3044.

5. Emery JD, Shaw K, Williams B, et al. The role of primary care in early detection and follow-up of cancer. Nat Rev Clin Oncol 2014; 11(1): 38-48.

6. Guldbrandt LM, Fenger-Gron M, Folkersen $\mathrm{BH}$, et al. Reduced specialist time with direct computed tomography for suspected lung cancer in primary care. Danish Med J2013; 60(12): $A 4738$

7. Ingeman ML, Christensen MB, Bro F, et al. The Danish cancer pathway for patients with serious non-specific symptoms and signs of cancer - a cross-sectional study of patient characteristics and cancer probability. BMC Cancer 2015; 15: 421

8. Vedsted P, Olesen F. A differentiated approach to referrals from general practice to support early cancer diagnosis - the Danish threelegged strategy. Br J Cancer 2015; 112(Suppl 1): S65-69.

9. Emery J. Assessment of cancer risk in men and women. Br J Gen Pract 2013; 63(606): 4-5.

10. Chiang PP, Glance D, Walker J, et al. Implementing a QCancer risk tool into general practice consultations: an exploratory study using simulated consultations with Australian general practitioners. Br J Cancer 2015: 112(Suppl 1): S77-83.

11. Neal RD, Din NU, Hamilton W, et al. Comparison of cancer diagnostic intervals before and after implementation of NICE guidelines: analysis of data from the UK General Practice Research Database. Br J Cancer 2014; 110(3): 584-592.

12. Meechan D, Gildea C, Hollingworth L, et al. Variation in use of the 2-week referral pathway for suspected cancer: a cross-sectional analysis. Br J Gen Pract 2012; DOI: 10.3399/ bjgp12X654551.

13. Rose PW, Rubin G, Perera-Salazar R, et al. Explaining variation in cancer survival between 11 jurisdictions in the International Cancer Benchmarking Partnership: a primary care vignette survey. BMJ Open 2015; 5(5): e007212.

14. De Angelis R, Sant M, Coleman MP, et al. Cancer survival in Europe 1999-2007 by country and age: results of EUROCARE-5 - a population-based study. Lancet Oncology 2014 15(1): 23-34. 